\title{
Using a chimeric respiratory chain and EPR spectroscopy to determine the origin of semiquinone species previously assigned to mitochondrial complex I
}

\author{
John J. Wright ${ }^{1,2}$, Justin G. Fedor ${ }^{2}$, Judy Hirst ${ }^{2}$ and Maxie M. Roessler ${ }^{1,3^{*}}$ (D)
}

\begin{abstract}
Background: For decades, semiquinone intermediates have been suggested to play an essential role in catalysis by one of the most enigmatic proton-pumping enzymes, respiratory complex I, and different mechanisms have been proposed on their basis. However, the difficulty in investigating complex I semiquinones, due to the many different enzymes embedded in the inner mitochondrial membrane, has resulted in an ambiguous picture and no consensus.

Results: In this paper, we re-examine the highly debated origin of semiquinone species in mitochondrial membranes using a novel approach. Our combination of a semi-artificial chimeric respiratory chain with pulse EPR spectroscopy (HYSCORE) has enabled us to conclude, unambiguously and for the first time, that the majority of the semiquinones observed in mitochondrial membranes originate from complex III. We also identify a minor contribution from complex II.

Conclusions: We are unable to attribute any semiquinone signals unambiguously to complex I and, reconciling our observations with much of the previous literature, conclude that they are likely to have been misattributed to it. We note that, for this earlier work, the tools we have relied on here to deconvolute overlapping EPR signals were not available. Proposals for the mechanism of complex I based on the EPR signals of semiquinone species observed in mitochondrial membranes should thus be treated with caution until future work has succeeded in isolating any complex I semiquinone EPR spectroscopic signatures present.
\end{abstract}

Keywords: NADH:ubiquinone oxidoreductase, Respiratory complex I, Semiquinones, Electron paramagnetic resonance

\section{Background}

Respiratory complex I (NADH:ubiquinone oxidoreductase) is a crucial component of the mitochondrial electron transport chain and thus plays a central role in energy conversion and cellular metabolism [1-3]. Complex I catalyses the oxidation of NADH and the reduction of ubiquinone, coupled to the translocation of four protons

\footnotetext{
* Correspondence: m.roessler@imperial.ac.uk

'School of Biological and Chemical Sciences, Queen Mary University of London, Mile End Road, London E1 4NS, UK

${ }^{3}$ Department of Chemistry, Imperial College London, Molecular Sciences Research Hub, White City Campus, Wood Lane, London W12 OBZ, UK Full list of author information is available at the end of the article
}

across the inner mitochondrial membrane in a mechanism that remains enigmatic, despite recent structural advancements [4]. The current consensus is that the binding and reduction of ubiquinone form an important mechanistic element, but while mechanisms for the long-range coupling that links electron transfer to proton translocation have been proposed on the basis of computational models [5-7], direct experimental evidence for them is lacking.

The key ubiquinone reduction step in catalysis must proceed via a semiquinone (SQ) radical, given that the iron-sulphur (Fe-S) clusters linking the two substrate binding sites are single electron carriers. It is thus no

(c) The Author(s). 2020 Open Access This article is licensed under a Creative Commons Attribution 4.0 International License, which permits use, sharing, adaptation, distribution and reproduction in any medium or format, as long as you give appropriate credit to the original author(s) and the source, provide a link to the Creative Commons licence, and indicate if changes were made. The images or other third party material in this article are included in the article's Creative Commons licence, unless indicated otherwise in a credit line to the material. If material is not included in the article's Creative Commons licence and your intended use is not permitted by statutory regulation or exceeds the permitted use, you will need to obtain permission directly from the copyright holder. To view a copy of this licence, visit http://creativecommons.org/licenses/by/4.0/. The Creative Commons Public Domain Dedication waiver (http://creativecommons.org/publicdomain/zero/1.0/) applies to the data made available in this article, unless otherwise stated in a credit line to the data. 
surprise that, over the past four decades, electron paramagnetic resonance (EPR) spectroscopy has been used extensively to investigate the SQ species associated with complex I. However, despite numerous reports of SQ catalytic intermediates forming during turnover [8-12], fundamental disagreements and inconsistencies have led to ambiguity about the importance of SQs in the energy coupling reaction of complex I [13]. Indeed, the stability of the SQs generated during turnover has recently been questioned [14], suggesting that SQs formed do not, in fact, accumulate sufficiently to enable them to be trapped and observed on an experimentally accessible timescale. Given that different mechanisms have been proposed on the basis of different ubiquinone reduction pathways [15-17], a re-investigation of the putative SQs associated with complex I is now warranted.

Most studies of complex I SQs have been conducted in mammalian submitochondrial particles (SMPs), inverted membrane vesicles that both maintain the structural integrity of the inner mitochondrial membrane and allow the NADH substrate to be provided directly for complex I catalysis in vitro. However, SMPs contain the entire complement of inner membrane enzymes (including many enzymes not directly associated with the electron transfer chain) and so any EPR signals observed are difficult to assign to specific enzymes. Assignments of SQs have relied on (1) the inhibition of enzymes downstream of complex I, removing SQs from their sites but invoking secondary effects through inhibition of turnover and alteration of the redox status of the Q-pool, and (2) an exogenous ubiquinone analogue $\left(\mathrm{Q}_{1}\right)[10,11]$, which has subsequently been shown to also react adventitiously at the complex I flavin (NADH binding) site [18]. Robust investigations of complex I SQs thus require sustained turnover conditions that both replenish the Q-pool and rely on only the natural, highly hydrophobic ubiquinone substrate.

Two SQs are typically attributed to complex I in SMPs, distinguished on the basis of their relaxation properties obtained from EPR measurements: fastrelaxing $\left(\mathrm{SQ}_{\mathrm{Nf}}\right)$ and slow-relaxing $\left(\mathrm{SQ}_{\mathrm{Ns}}\right)$ [10, 11$]$. $\mathrm{SQ}_{\mathrm{Nf}}$ is reported to form only when a substantial proton-motive force $(\Delta \mathrm{p})$ is present across the inner mitochondrial membrane [10], and to interact with the nearby terminal Fe-S cluster (N2) $[8,19]$. However, very particular conditions have been reported to be required for the clear observation of $\mathrm{SQ}_{\mathrm{Nf}}$ and the SQ-N2 interaction. $\mathrm{SQ}_{\mathrm{Ns}}$ has been reported in SMPs in the absence of $\Delta \mathrm{p}$ [9] and assigned to complex I based on its sensitivity to complex I Q-site inhibitors, and because it is absent when complex $\mathrm{I}$ is in the deactive state [8]. Notably, neither signal has been clearly observed in any purified enzyme system, so that their assignments to complex I have never been proven. Investigations using complex I in proteoliposomes $[12,20]$ have only provided much lower SQ signal intensities and occupancies than observed in SMPs: $<0.1 \mu \mathrm{M}$ SQ species and $\sim 2 \%$ total occupancy [12], compared to $\sim 2.5 \mu \mathrm{M}$ and $\sim 100 \%$ occupancy [10] in SMPs. The lack of clear evidence for the assignment of observed SQ species to complex I in SMPs demands further investigation into their origins.

Here, we used the hitherto-unexploited approach of constructing a chimeric respiratory chain from SMPs and an alternative ubiquinol oxidase, to enable targeted inhibition of enzymes downstream of complex I while maintaining its turnover with the native ubiquinone substrate (Fig. 1). This approach circumvents the misleading effects resulting from soluble quinone analogues, and allows the contributions of SQs from other respiratory chain elements to be probed. Although a chimeric respiratory chain using SMPs and the purified cytochrome bd complex from Escherichia coli has been described previously [21], it is unsuitable for SQ studies owing to the very stable and therefore dominant cytochrome $b d$ SQ [22, 23]. Addition of the alternative oxidase (AOX) from Trypanosoma brucei brucei to SMPs creates 'AOX-SMPs', containing a quinone-cycling system between complex I and AOX and with NADH: $\mathrm{O}_{2}$ turnover sensitive to complex I inhibitors (such as piericidin $\mathrm{A}$ and rotenone) and the AOX inhibitor ascofuranone [24, 25]. As no SQ intermediates have been detected by EPR spectroscopy in wild-type AOX [26, 27], incorporation of this terminal oxidase into SMPs represents an intriguing new platform to investigate the SQs that are generated during sustained $\mathrm{NADH}$ oxidation by complex I.

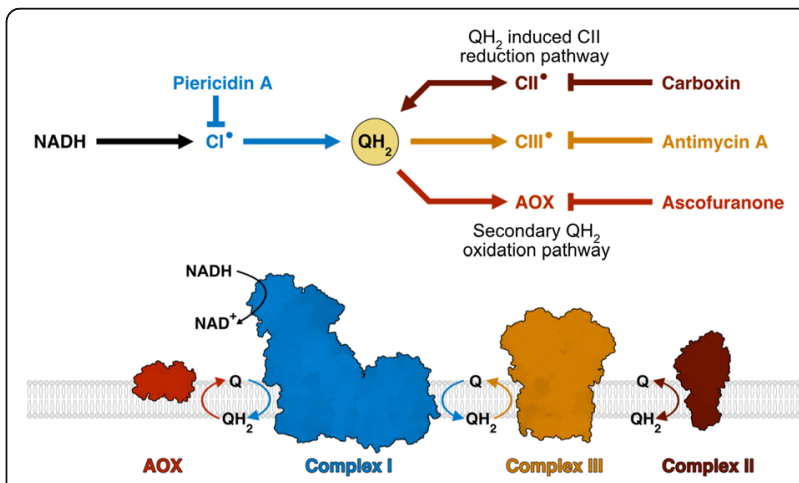

Fig. 1 Quinone cycling in AOX-SMPs during NADH oxidation. (Top) Flow diagram showing the routes of quinol $\left(\mathrm{QH}_{2}\right)$ production and oxidation and their inhibitor sensitivities. Radicals (•) indicate expected formation of semiquinones. (Bottom) AOX-SMPs support $\mathrm{NADH}: \mathrm{O}_{2}$ turnover through both the canonical chain and the complex I-AOX pathway 


\section{Results}

\section{SMPs and AOX-SMPs are well coupled and sustain a} proton-motive force

The variation in SQs observed previously in different preparations of SMPs demands careful characterisation of our membrane system. The SMPs used here (see $[28,29]$ ) are capable of NADH- and succinatedriven ATP synthesis and ATP hydrolysis and are intrinsically well coupled without additional coupling factors, so they are a physiologically relevant system for studying SQ formation. The addition of AOX provides a rapid secondary quinol oxidation route (Fig. 1), such that $\mathrm{NADH}: \mathrm{O}_{2}$ turnover is capable of exceeding turnover by the canonical respiratory chain in an AOX-concentration dependent manner [24, 25]. Thus, to prevent the substrate from becoming exhausted and ensure turnover during EPR sample preparation, AOX was titrated to a low level, at which the flux through the complex I-AOX pathway matched that through the canonical chain (Additional file 1, Figure S1). Representative catalytic properties for the original SMPs and the rate-matched AOX-SMPs are given in Table 1. In line with previous studies [28, 29], RCR values of 1.6 to 3.0 were typically observed in the absence of $\mathrm{AOX}$, and substantial rates of $\mathrm{NAD}^{+}$reduction (reverse electron transfer, RET, driven by a reduced Q-pool formed by succinate oxidation and a proton-motive force $(\Delta \mathrm{p})$ formed by ATP hydrolysis) were observed in both cases (Table 1). The value of $\Delta \mathrm{p}$ established by ATP hydrolysis $(\sim 160 \mathrm{mV}$, Table 1$)$ was determined by varying the potential of NADH:fumarate oxidoreduction, and matches previously reported values $[24,28]$. Therefore, the loss of the RCR in AOX-SMPs does not arise from a loss of coupling, since the $\Delta \mathrm{p}$ supported by ATP hydrolysis is shown to be substantial and near identical to that in the original SMPs.

The proton-pumping capacity of AOX-SMPs was further visualised through quenching of ACMA fluorescence during $\mathrm{NADH}: \mathrm{O}_{2}$ turnover (Fig. 2). Substantial quenching was observed in AOX-SMPs upon addition of NADH. The addition of antimycin A (either before or after NADH addition) causes a partial ablation of the ACMA fluorescence quenching due to continued catalysis and proton pumping by complex I. A combination of

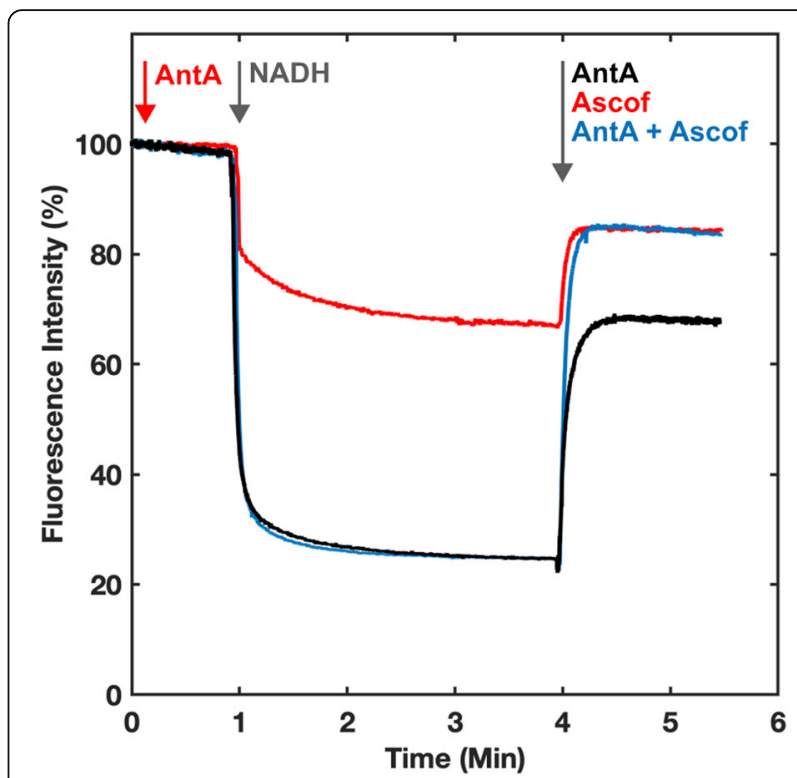

Fig. 2 The effect of oxidase pathway inhibitors on quenching ACMA fluorescence in AOX-SMPs. ACMA fluorescence quenching was measured (excitation at $419 \mathrm{~nm}$, emission at $484 \mathrm{~nm}$ ) during $\mathrm{NADH}: \mathrm{O}_{2}$ turnover in AOX-SMPs. The rate of coupled NADH oxidation in AOX-SMPs was rate-matched as in Additional file 1, Figure S1. Measurements were performed with constant stirring at $32^{\circ} \mathrm{C}$ in a buffer containing $10 \mathrm{mM}$ Tris- $\mathrm{SO}_{4}, 50 \mathrm{mM} \mathrm{KCl}$. In total, $50 \mu \mathrm{g} / \mathrm{mL}$ of SMPs or AOX-SMPs were added to a mixture containing $0.5 \mu \mathrm{M}$ ACMA and $0.1 \mu \mathrm{M}$ valinomycin. NADH was added to $500 \mu \mathrm{M}$. Turnover was inhibited by addition of antimycin A ( $2 \mu \mathrm{M})$ and/or ascofuranone $(2 \mu \mathrm{M})$ as indicated

antimycin A and the specific AOX inhibitor ascofuranone fully ablates quenching. Following prior incubation with antimycin A, the level of ACMA quenching from complex I turnover is approximately $30-40 \%$ of the quenching without any inhibitor, as expected for the rate-matched AOX-SMPs from the established stoichiometry [29]. Quenching was fully sensitive to complex I inhibition by piercidin $\mathrm{A}$, as well as to uncoupling to dissipate $\Delta \mathrm{p}$ (Additional file 2, Figure S2). Levels of ACMA quenching were identical in both SMPs and AOX-SMPs (Additional file 2, Figure S2) in the absence of inhibitors, and ACMA quenching by SMPs and AOX-SMP during ATP hydrolysis matches closely (Additional file 2, Figure S2). This further demonstrates that the coupling of the membrane is retained in the AOX system.

Table 1 Representative kinetic measurements on SMPs and AOX-SMPs. AOX-SMPs were preincubated with antimycin A. For assay details, see "Methods"

\begin{tabular}{|c|c|c|c|c|c|c|c|c|}
\hline & \multicolumn{3}{|c|}{$\mathrm{NADH}: \mathrm{O}_{2}\left(\mu \mathrm{mol} \mathrm{min}^{-1} \mathrm{mg}^{-1}\right)$} & \multicolumn{2}{|c|}{ Succinate:NAD $(\mathrm{RET})\left(\mu \mathrm{mol} \mathrm{min}^{-1} \mathrm{mg}^{-1}\right)$} & \multicolumn{2}{|c|}{ Inhibition of NADH oxidation (\%) } & \multirow[t]{2}{*}{$\Delta p(m V)$} \\
\hline & -Gram & + Gram & $\mathrm{RCR}$ & -Gram & +Gram & Piericidin A & Quinol oxidation $^{\mathrm{a}}$ & \\
\hline SMPs & $0.301 \pm 0.008$ & $0.626 \pm 0.011$ & $2.08 \pm 0.05$ & $0.092 \pm 0.004$ & $0.009 \pm 0.001$ & $98.7 \pm 0.01$ & $95.8 \pm 0.03$ & $161.4 \pm 0.2$ \\
\hline AOX-SMPs & $0.288 \pm 0.026$ & $0.360 \pm 0.028$ & $1.25 \pm 0.03$ & $0.065 \pm 0.004$ & $0.007 \pm 0.005$ & $99.2 \pm 0.01$ & $97.1 \pm 0.01$ & $157.9 \pm 1.6$ \\
\hline
\end{tabular}

${ }^{\mathrm{a}}$ Quinol oxidation was inhibited by antimycin A in SMPs, and antimycin A and ascofuranone in AOX-SMPs 
The $g \sim 2 \mathrm{EPR}$ signal in the presence of $\mathrm{O}_{2}$ in SMPs In agreement with previous work [30], a large radical signal is observed at $g \sim 2$ (Fig. 3) upon addition of $\mathrm{NADH}$ to SMPs supplemented with additional $\mathrm{O}_{2}$. This signal is almost negligible in SMPs not supplemented with $\mathrm{O}_{2}$ (Additional file 3, Figure S3), and it is typically attributed to SQs arising from the respiratory chain complexes. The relatively narrow linewidth ( $9 \mathrm{G})$ defines the signal as arising from one or more SQ species, rather than a complex I flavosemiquinone, as this would exhibit a broader linewidth (>15 G) [31, 32]. The other EPR signals are primarily due to Fe-S clusters in complex I (referred to using the $\mathrm{Nx}$ nomenclature) and the other respiratory complexes, which are predominantly reduced during turnover. Due to their faster relaxation compared to the $g \sim 2$ radical signal, which makes the radical signal stand out more at lower power $(0.02 \mathrm{~mW})$, the $\mathrm{Fe}-\mathrm{S}$ signals are best observed at lower temperature (16 K). At higher temperature $(40 \mathrm{~K})$, the slowest-relaxing complex I [2Fe-2S] cluster N1b dominates the Fe-S region of the spectrum, alongside the prominent $g \sim 2$ signal. The broad dip in the same region from the oxidised $\mathrm{Cu}_{\mathrm{a}}$ centre of complex IV (most prominently observed at $16 \mathrm{~K}$ owing to the fast relaxation of the $\mathrm{Cu}_{\mathrm{a}}$ centre) confirms the sustained turnover condition [33] in the presence of $\mathrm{O}_{2}$.

\section{Assignment of the $g \sim 2$ EPR signal through inhibition studies}

To assign the origin of the prominent $g \sim 2$ EPR signal, a series of AOX-SMP samples were prepared containing different inhibitors (Fig. 4). AOX-SMP samples were chosen rather than SMP samples because turnover in the presence of inhibitors can only be sustained by employing the chimeric respiratory chain (Fig. 1). Akin to what is observed for SMPs, the $g \sim 2$ EPR signal increases substantially in intensity in the presence of additional $\mathrm{O}_{2}$ (Fig. 4A, B). Note that all spectra in Fig. 4 were recorded at a high microwave power $(2.02 \mathrm{~mW})$ to use the (fast-relaxing) Fe-S cluster signals for reference. Carboxin, a complex II inhibitor, ablated a contribution observed most clearly at $g=1.99$ (more clearly seen at $12 \mathrm{~K}$, see Fig. 5) that results from a well-documented SQ-SQ radical pair in the complex II Q-site $[34,35]$ and is a consequence of the highly reduced Q-pool during $\mathrm{NADH}: \mathrm{O}_{2}$ turnover (Fig. 1). However, carboxin had little effect on the overall $g \sim 2$ signal (compare Fig. 4B, C and see Additional file 4, Figure S4 for the dependence of signal intensity on microwave power). Antimycin A, a

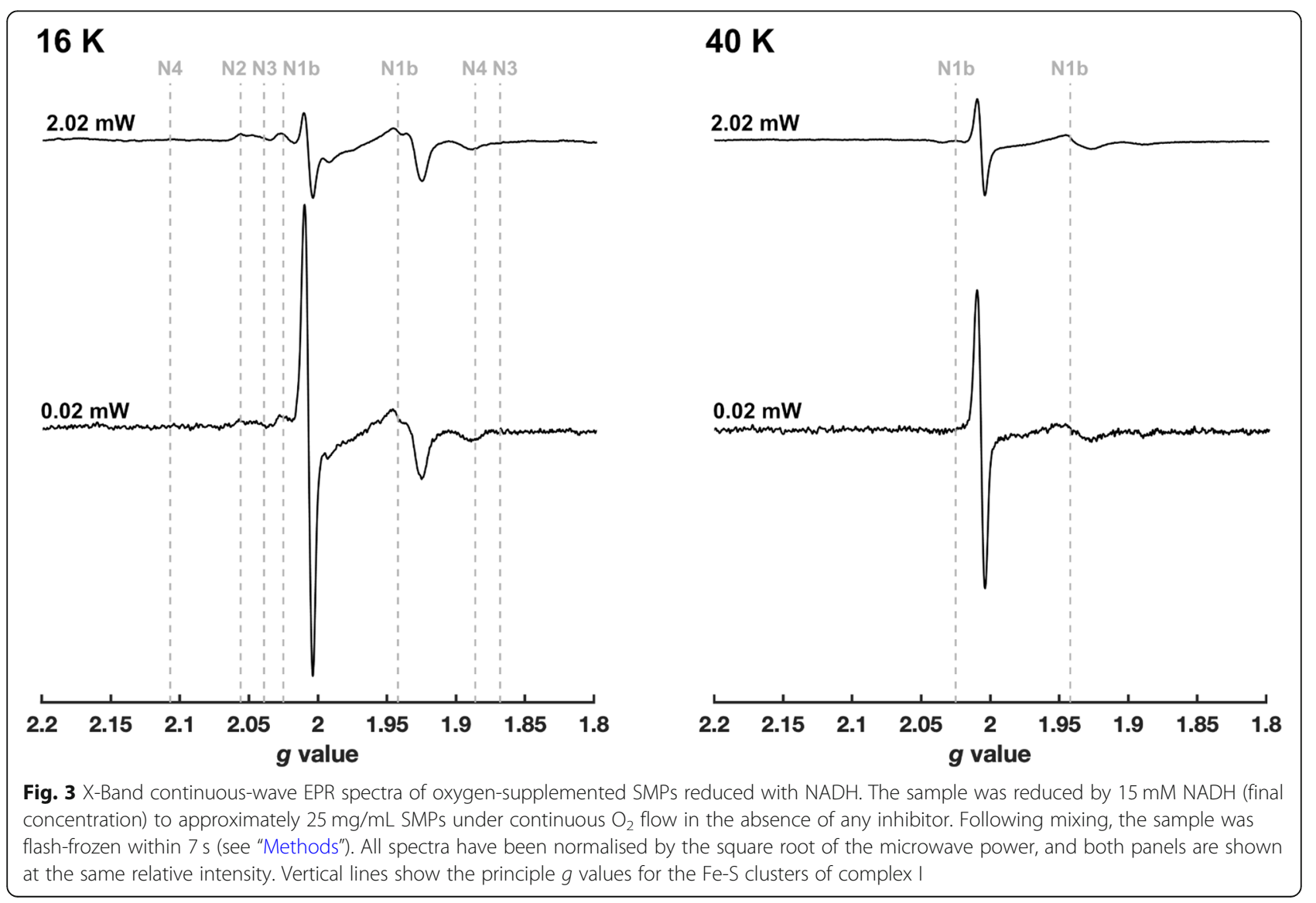




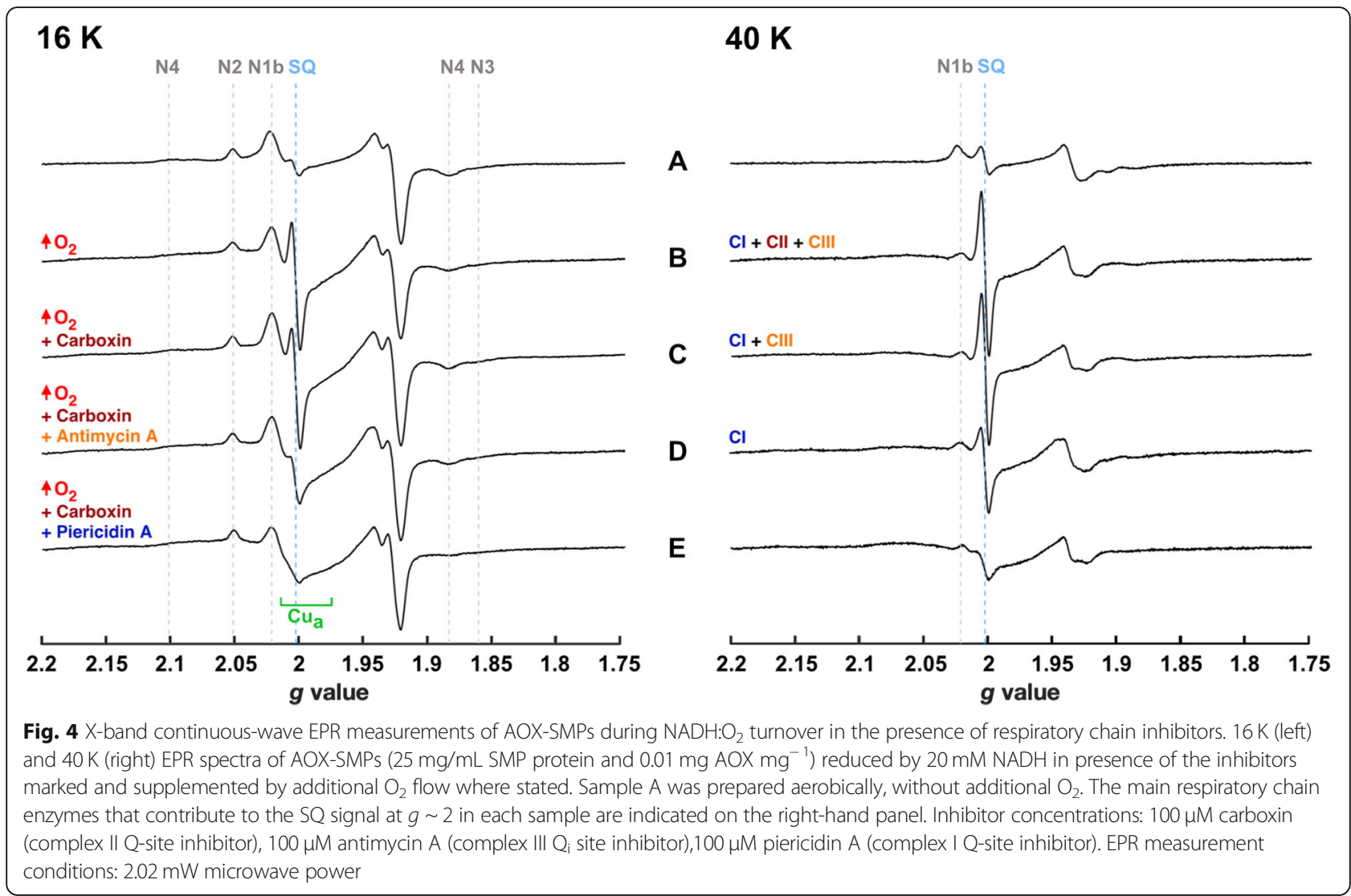

complex III $\mathrm{Q}_{\mathrm{i}}$ site inhibitor, diminished the $g \sim 2$ signal intensity substantially (Fig. 4D). The major SQ species present during $\mathrm{NADH}$ oxidation (note that complex I turnover continues in the presence of antimycin A, due to the presence of AOX) must therefore arise from complex III. Finally, the complex I specific Q-site inhibitor piericidin A effectively eliminates the $g \sim 2$ signal (Fig. 4E) because it prevents quinone reduction and SQ radicals forming anywhere in the chain. Therefore, the $g$ $\sim 2$ signal comprises (at least) three distinct SQ species: the major contribution is from complex III, there is a lesser contribution from complex II, and a further minor contribution (revealed by comparison of Fig. 4D and E) that may be, but is not proven to be, from complex I.

\section{Exploiting sustained turnover to investigate the possible complex I SQ}

Being as the AOX-SMP system enables steady-state complex I turnover independently of complex III, we reasoned that any $g \sim 2$ SQ signals from complex I in the presence of NADH (with complexes II and III inhibited) should be retained in AOX-SMPs (complex I is turning over) but not in SMPs (complex I is not turning over). Figure 6 shows that the $g \sim 2$ signal observed is larger in AOX-SMPs compared to SMPs (the increase is small but highly consistent) and that it is amplified over time but does not increase further with prior activation of complex I. The relative increase in the $g \sim 2$ signal in AOXSMPs over time is thus unlikely to result from activation of complex I and could result from evolution of the oxidation state of the ubiquinone pool. However, the presence of any $g \sim 2$ signal in SMPs that are not undergoing turnover is surprising: complex II/III-inhibited SMPs should emulate the anaerobic condition in which the ubiquinone pool simply becomes fully reduced by NADH (Fig. 6, top spectrum), and therefore the $g \sim 2$ signal intensity is expected to be insignificant. The origin of the $g \sim 2$ signal in Fig. 6, which thus appears to be stabilised or induced by the presence of $\mathrm{O}_{2}$, is therefore unclear. Its sensitivity to piericidin $A$ in AOX-SMPs (Fig. 4E) make it tempting to assign it to complex I, but its presence in SMPs inhibited with antimycin A means that downstream effects mediated by changes in the potential of the ubiquinone pool, perhaps in other Q-linked enzymes, cannot be excluded.

\section{Using hyperfine spectroscopy to distinguish overlapping SQ signals}

As X-band CW EPR spectroscopy cannot unambiguously distinguish different SQ species (their $g$ values and linewidths are too similar), we employed hyperfine spectroscopy to differentiate the SQs on the basis of the surrounding nuclear spins $\left({ }^{14} \mathrm{~N}, I=1\right)$ coupled to the 


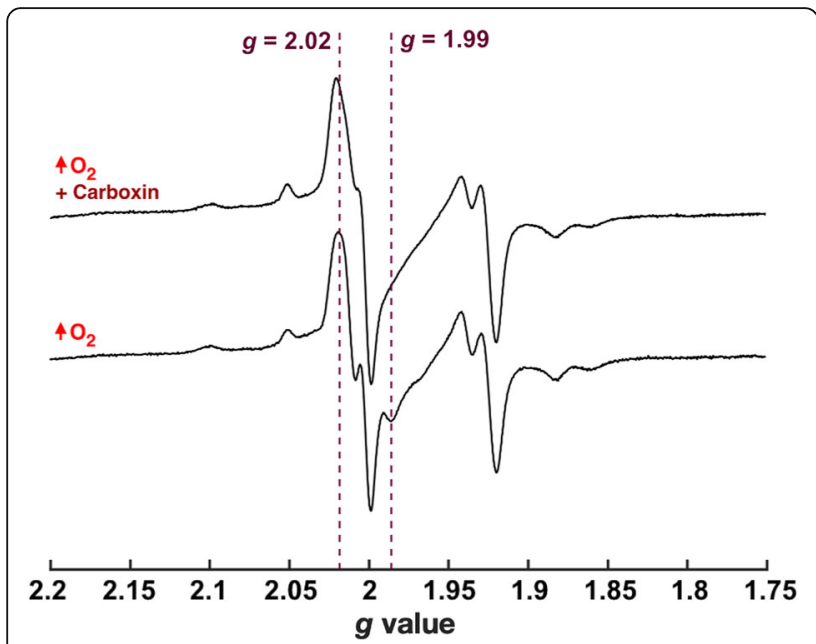

Fig. 5 The effect of carboxin on complex II semiquinone signals at $12 \mathrm{~K}$. AOX-SMPs were prepared and measured as in Fig. 4 but at 12 $K$. Vertical lines indicate $g$ values for the SQ-SQ radical pair of complex II as previously reported [34]

electron spin. Equivalent but more concentrated $(\sim 2 \mathrm{x})$ samples than those shown in Fig. 4 were prepared for HYSCORE spectroscopy. The ${ }^{14} \mathrm{~N}$ regions of the $40 \mathrm{~K}$ HYSCORE spectra of carboxin-treated, and carboxinand antimycin A-treated samples are shown in Fig. 7.

Whereas the spectrum from the complex II-inhibited sample exhibits two sets of ${ }^{14} \mathrm{~N}$ cross peaks at [3.1, 4.3] MHz and [3.0, 2.1] MHz (Fig. 7a), the latter peaks are absent when both complex II and III are inhibited (Fig. 7b). Indeed, the HYSCORE difference spectrum reveals a single set of ${ }^{14} \mathrm{~N}$ cross peaks (Fig. 7c) that could be simulated (Fig. 7d) with hyperfine and quadrupolar parameters that are highly consistent with those determined for the strongly $\mathrm{H}$-bonded $\mathrm{Q}_{\mathrm{i}}$ site $\mathrm{SQ}$ radical in complex III isolated from Rhodobacter sphaeroides which forms an interaction with the $\mathrm{N}_{\varepsilon}$ of a histidine (Additional file 6, Table S1) [36]. Therefore, the HYSCORE measurements enable unambiguous assignment of the antimycin A-sensitive SQ species to complex III, consistent with the conclusion from CW measurements (Fig. 4) that the major SQ radical stabilised in SMPs during NADH: $\mathrm{O}_{2}$ turnover arises from complex III. The complete simulation of the ${ }^{14} \mathrm{~N}$ environment of the SQ in carboxin-inhibited SMPs is given in Fig. 8a.

The quadrupole coupling parameters $(\kappa$ and $\eta$-see Eq. 3) of ${ }^{14} \mathrm{~N}$ nuclei can be highly informative as to the type of nitrogen interacting with the electron spin (Fig. 8b). The prominent set of cross peaks at [3.1, 4.3] $\mathrm{MHz}$ present in both samples could be simulated well with hyperfine and quadrupole parameters that are typical of backbone amide ${ }^{14} \mathrm{~N}$ (Fig. 8a, see also Additional file 6 , Table $\mathrm{S} 1$ ), as revealed by comparison with the ${ }^{14} \mathrm{~N}$ quadrupole parameters of known protein-bound

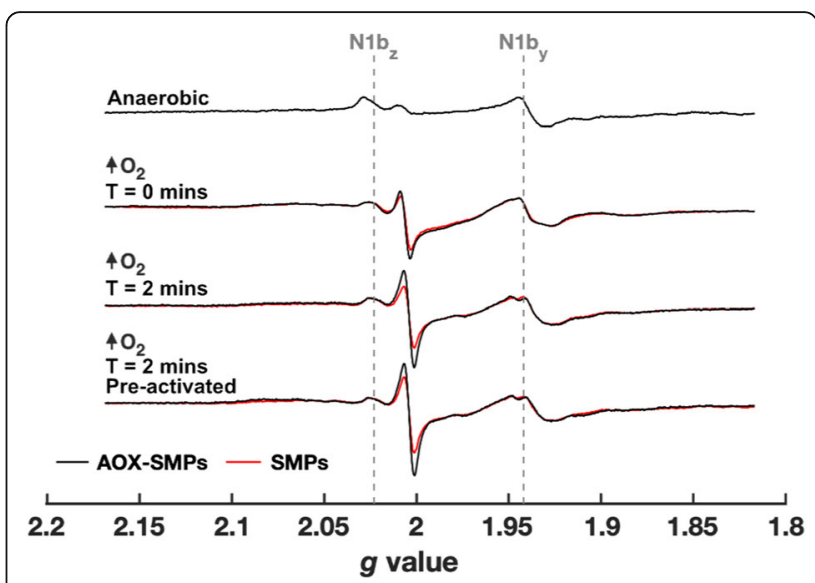

Fig. 6 Comparison of SQ EPR signals in antimycin A- and carboxininhibited SMPs and AOX-SMPs. X-band EPR spectra of SMPs (red)

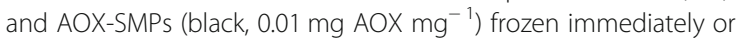
following a 2-min incubation with $40 \mathrm{mM}$ NADH under high $\mathrm{O}_{2}$ flow. Samples were treated with $100 \mu \mathrm{M}$ carboxin and $100 \mu \mathrm{M}$ antimycin A. Pre-activated samples were mixed with $1 \mathrm{mM} \mathrm{NADH}$ and incubated at room temperature for $5 \mathrm{~min}$ in an aerobic atmosphere prior to EPR sample preparation. The anaerobic AOXSMP sample was incubated in a Braun UniLab-plus glovebox $\left(\mathrm{O}_{2}<\right.$ $0.5 \mathrm{ppm}, \mathrm{N}_{2}$ atmosphere) at room temperature $\left(22^{\circ} \mathrm{C}\right)$ for $1 \mathrm{~h}$ prior to addition of $20 \mathrm{mM} \mathrm{NADH}$. The sample was frozen within $30 \mathrm{~s}$ of substrate mixing. EPR measurement conditions: $2.02 \mathrm{~mW}$ microwave power, $40 \mathrm{~K}$

SQs (Fig. 8b). Given that contributions from the Fe-S clusters (specifically N1b, see ref. [38]) are minimal relative to the contributions from the $g \sim 2$ signal (shown by measurements conducted off-resonance from the $g \sim 2$ SQ signal, see Additional file 7, Figure S6 and Additional file 8, Figure S7), these ${ }^{14} \mathrm{~N}$ modulations must originate from a SQ radical. It is tempting to attribute the cross peaks to complex I SQ (given that complex II is inhibited in both samples), but their generic spectroscopic signature necessitates caution and a definitive assignment cannot be made.

\section{Discussion}

The AOX-SMPs used here have enabled us, for the first time, to investigate the effects of respiratory chain inhibitors on SQ species formed downstream of complex I, while sustaining complex I turnover with its native ubiquinone substrate. Many previous studies into complex I SQs have refrained from using inhibitors due to emphasis on the requirement for sustained turnover $[8,30,39]$. However, SQs can be stabilised by complexes I, II [35, 40, 41] and III [36, 42, 43], as well as by many other candidate off-pathway enzymes, so inhibitors have a crucial part to play in dissecting their origins. Inhibitors have been combined with NADH: $\mathrm{Q}_{1}$ turnover, but $\mathrm{Q}_{1}$ and other soluble ubiquinone analogues are known to react at the complex I flavin site through SQ 


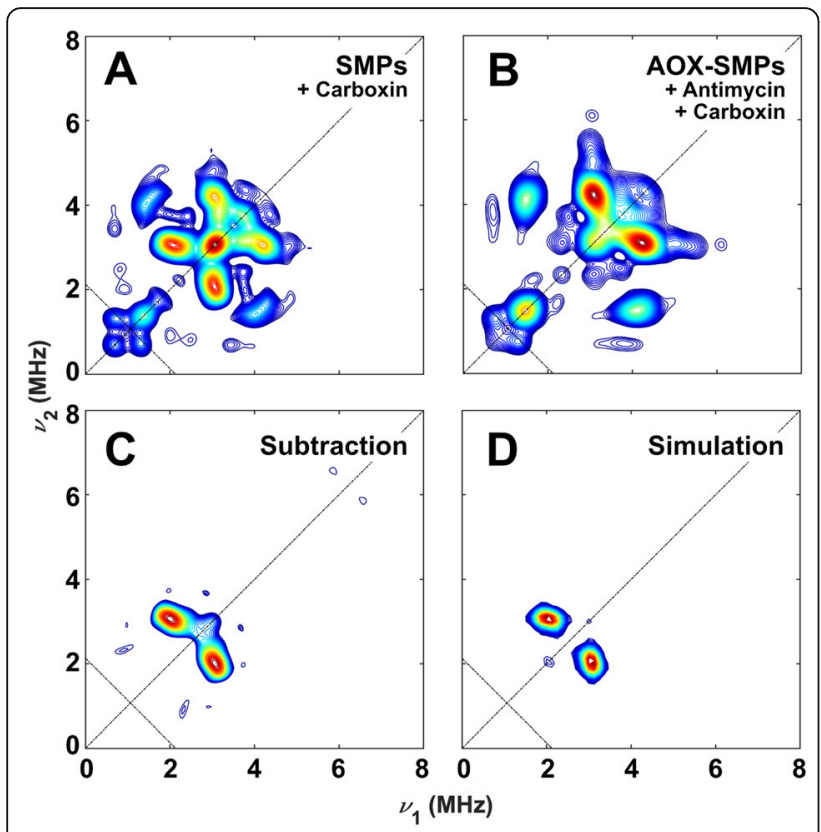

Fig. 7 Comparison of ${ }^{14} \mathrm{~N}$ couplings in HYSCORE spectra of SMPs and AOX-SMPs at $40 \mathrm{~K}$. X-band spectra (a and $\mathbf{b}$ ) were recorded at $g=2.006$ with a four-pulse sequence $\left(\pi / 2-\tau-\pi / 2-t_{1}-\pi-t_{2}-\pi / 2-\tau-\right.$ echo) with $\pi / 2=16 \mathrm{~ns}, \pi=16 \mathrm{~ns}, \tau=116 \mathrm{~ns}$, shot repetition time 2 $\mathrm{ms}$. The intensity of the stimulated echo (c) was normalised to the intensity of the cross peaks at $[4.1,1.6] \mathrm{MHz}$. Simulations (d) were calculated as a single ${ }^{14} \mathrm{~N}$ nucleus coupled to a single electron spin with parameters $A_{\text {iso }}=0.54 \mathrm{MHz}, T=-0.2 \mathrm{MHz}, k=0.425 \mathrm{MHz}, \eta=$ $0.18 \mathrm{MHz}$ (see Eq. 3 for calculation of quadrupole coupling parameters). Antidiagonal lines are given for the ${ }^{14} \mathrm{~N}$ Larmor frequency. Very similar data as in a were recorded for AOX-SMPs in the presence of carboxin (Additional file 5, Figure S5); the data shown here are for SMPs because of their higher signal to noise ratio. No strongly coupled ${ }^{14} \mathrm{~N}$ nuclei were detected in the $(-,+)$ quadrant; for the entire $(+,+)$ quadrant (including the ${ }^{1} \mathrm{H}$ region), see Figures S6 and S7 in Additional files 7 and 8, respectively

intermediates in potentially misleading side reactions [18]. Our chimeric respiratory chain supports sustained complex I turnover while avoiding these side reactions. Furthermore, the membrane particles used here show substantial proton-pumping capacity (without induced coupling' by oligomycin or other poorly characterised treatments) as demonstrated by ACMA fluorescence and $\Delta \mathrm{p}$ measurements, and the AOX-SMP system has recently been verified as a physiologically relevant model for oxidative phosphorylation, with substantial rates of ATP synthesis and confirmed proton-pumping stoichiometries [24].

The majority of studies of SQ radicals in SMPs have used deconvolution of power saturation curves to determine the number of SQ species present, along with their relative relaxation rates. However, power saturation curves are prone to overinterpretation, with better fits to a single curve obtained by increasing the number of independently variable parameters [13], and they are unable to localise species to any particular respiratory chain component. Here we have used, for the first time, pulse EPR methods to investigate the origin of SQs in SMPs-methods that were not available when the first investigations were carried out some 40 years ago. HYSCORE measurements, combined with the modular AOX-SMP system, allowed us to spectroscopically isolate the $\mathrm{Q}_{i}$ site $\mathrm{SQ}$ in mammalian complex III for the first time, providing details of specific nitrogen hydrogen bonding interactions that closely resemble those in its purple bacterial counterpart [44]. Figure 7 shows that the quadrupolar parameters from the $\mathrm{H}$-bonded complex III $\mathrm{SQ}_{\mathrm{i}}$ are distinctive, strongly supporting our assignment. Recent work on the complex III SQ allows us to further qualify the type of complex III SQ we are observing. First, we consider the recently discovered $Q_{o}$ site metastable SQ [45] to be an unlikely contributor to the complex III $g=2.00$ signal because $Q_{o}$ site SQs have not been reported under steady-state aerobic conditions in SMPs as employed here [43]. Two populations of SQ are in principle possible in the $\mathrm{Q}_{\mathrm{i}}$ site, one with a dipolar coupling interaction to the neighbouring oxidised (paramagnetic) heme $b_{\mathrm{H}}$ and another without magnetic coupling to the reduced (diamagnetic) state of the heme [46]. The magnetically coupled $\mathrm{Q}_{\mathrm{i}}$ site $\mathrm{SQ}$ is not a contributor under the measurement conditions employed here because its enhanced spin-lattice relaxation means that it is only detectable at high temperature $(200 \mathrm{~K})$ and microwave power $[44,46]$. The slower relaxing uncoupled $Q_{i}$ site $S Q$ on the other hand can be formed during the oxidation of ubiquinol at the $\mathrm{Q}_{\mathrm{i}}$ site and is fully compatible with the spectroscopic properties of the $\mathrm{Q}_{\mathrm{i}}$ site semiquinone generated here in SMPs.

Comparison of our observed CW EPR spectra with known spectra from a radical pair in complex II (Fig. 5), and our HYSCORE spectra with known spectra from SQ in the complex III $\mathrm{Q}_{i}$ site (Fig. 7), has thus allowed most of the SQ signal observed during NADH oxidation in SMPs to be assigned. Quantification of the SQ signal in the $\mathrm{O}_{2}$-rich samples shown in Fig. 4 gave estimated values of $\sim 52 \%$ occupancy (SQ per N2), decreasing to $41 \%$ in the presence of carboxin, and to $\sim 15 \%$ in the presence of both antimycin A and carboxin, indicating that complex III makes the largest known contribution, followed by complex II. Thus, while the aim of this study was to characterise the $\mathrm{SQ}(\mathrm{s})$ present in complex I, only limited and ambiguous evidence has been obtained for these species. A minor, piericidin A-sensitive signal, observed under high $\mathrm{O}_{2}$ partial pressures in AOX-SMPs and SMPs in the presence of carboxin and antimycin A may arise from complex I turnover-but it may also arise from any other enzyme linked to the reduced ubiquinone pool. Since this signal (or, at least, a contribution to this signal) is observed in antimycin A-inhibited 

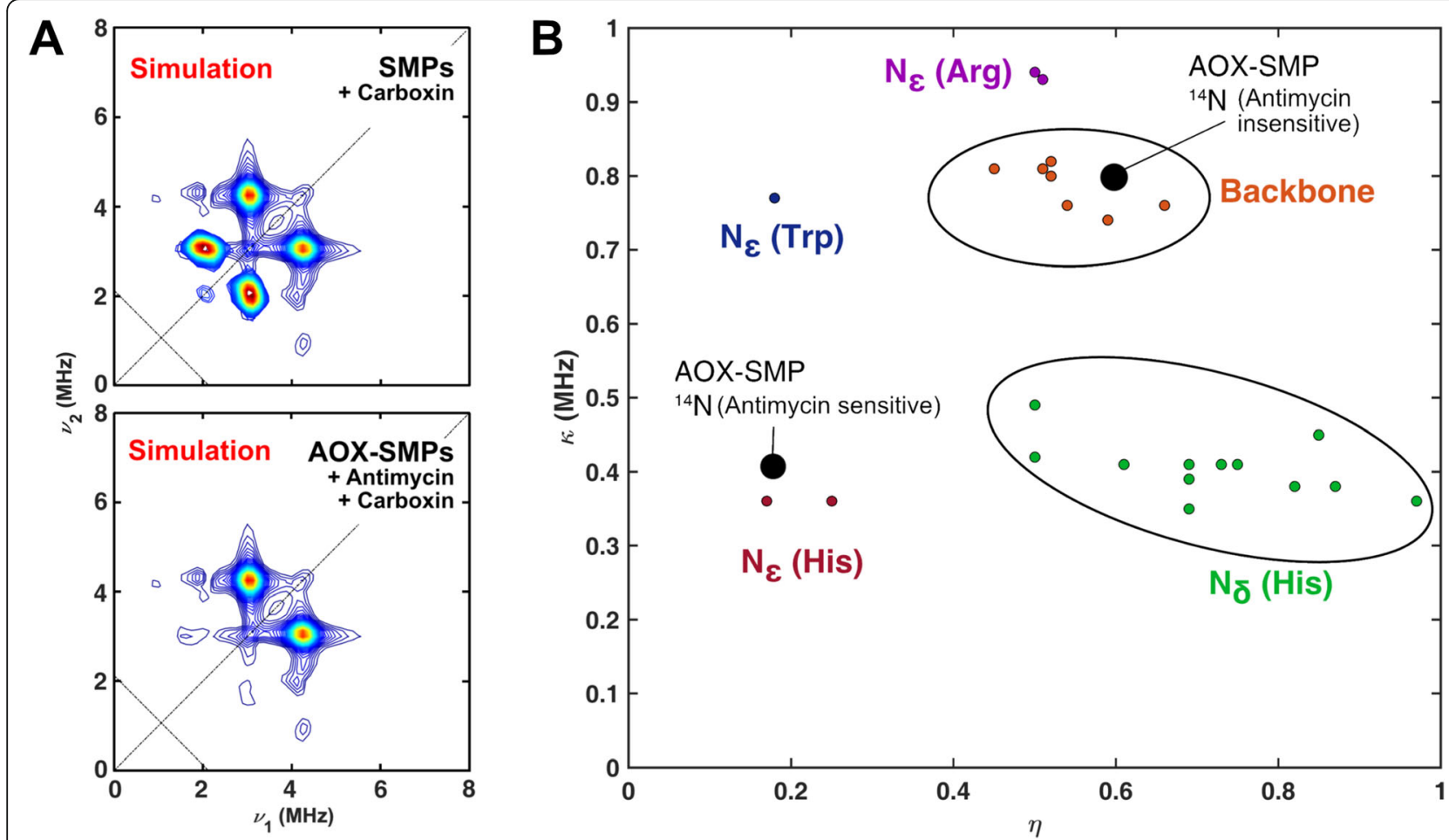

Fig. 8 Analysis of ${ }^{14} \mathrm{~N}$ couplings from antimycin A-inhibited AOX-SMPs: Quadrupole coupling parameters indicate the identity of ${ }^{14} \mathrm{~N}$ nuclei. a Simulated HYSCORE spectrum of SMPs inhibited with carboxin and AOX-SMPs inhibited with carboxin and antimycin. Simulation of major ${ }^{14} \mathrm{~N}$ cross peaks with $A_{\text {iso }}=1.00 \mathrm{MHz}, T=0.08 \mathrm{MHz}, \mathrm{K}=0.8 \mathrm{MHz}, \eta=0.6 \mathrm{MHz}$ (Antimycin insensitive) and $A_{\text {iso }}=0.54 \mathrm{MHz}, T=-0.2 \mathrm{MHz}, \mathrm{K}=0.425 \mathrm{MHz}$, $\eta=0.18 \mathrm{MHz}$ (Antimycin sensitive). ${ }^{14} \mathrm{~N}$ cross peaks were simulated as two electron spins each coupled to a single ${ }^{14} \mathrm{~N}$ nucleus. See methods (Eq. 3) for quadrupole coupling parameters. b Reported ${ }^{14} \mathrm{~N}$ quadruple coupling parameters for protein-bound SQs. The two observed sets of ${ }^{14} \mathrm{~N}$ couplings from AOX-SMP are labelled on the basis of their antimycin A sensitivity (both species are carboxin insensitive). b was adapted from [37]

SMPs (that have no oxidase activity, Fig. 6), complex I turnover is not required for its presence-but nonetheless, it is absent when complex I is reduced by $\mathrm{NADH}$ under anoxic conditions (Fig. 6), which should also reduce any other ubiquinone-linked enzyme. Therefore, the results suggest that the signal arises from an interaction with $\mathrm{O}_{2}$, under the high $\mathrm{O}_{2}$ partial pressures used here to sustain turnover. Non-catalytic oxidation of ubiquinol itself, or of the cofactors in complex I and other enzymes, may allow the slow turnover of complex I, or $\mathrm{O}_{2}$ may interact directly with bound quinones in complex I or other enzymes to generate SQ species. The increase of the $g \sim 2$ signal observed in AOX-SMPs (Fig. 6) may result from an increased steady-state concentration of complex I SQs, but this interpretation remains tentative. Alternatively, for example, AOXSMPs may harbour the increasing product of a catalytic side reaction that either involves AOX or depends strongly on the ubiquinone pool potential. Therefore, we are unable to definitively assign any of the $g \sim 2$ EPR signals observed to complex I.

Based on the demonstrable proton-pumping capacity of the SMP and AOX-SMP preparations used here, we expected that the $g_{\mathrm{z}}$ component of the N2 cluster should be split by magnetic interactions with complex I SQ $\left(\mathrm{SQ}_{\mathrm{Nf}}\right)$. This phenomenon was not observed. Changes in the region of the $\mathrm{N} 2 g_{\mathrm{z}}$ component were observed but required prolonged incubation with $\mathrm{NADH}$, and the N2 $g_{z}$ signal intensity was not affected (Additional file 9, Figure S8). Furthermore, they were concomitant with a decrease in the complex II S3 signal and so are unlikely to be associated with the N2 cluster of complex I. We also did not observe a profound reduction of SQ signal intensity at $16 \mathrm{~K}$ upon addition of uncouplers [8] (Additional file 9, Figure S8). Our results question the relevance of these features for complex I catalysis, although we cannot unambiguously exclude that they only appear under higher $\Delta \mathrm{p}$ values than were achieved here. While our AOX-SMPs show a lower RCR than those reporting to support $\mathrm{N} 2$ signal splitting, it is notable that when the NADH:Q ${ }_{1}$ assay system was used, the RCR value dropped to $1.2-1.5$ but N2 splitting and the presence of $\mathrm{SQ}_{\mathrm{Nf}}$ were still reported [11].

Previous investigations of SQs in SMPs have been inconsistent in their reasons for assigning SQs to complex I, rather than to complexes II and III. Early studies (which produced the most convincing SQ signal relative to the Fe-S spectrum of complex I) did not inhibit complex II or 
III to ablate their SQs $[8,47]$ so it is likely that the SQ signals they observed were dominated by enzymes downstream of complex I, particularly complex III. Subsequent studies, including those that relied on $\mathrm{NADH}: \mathrm{Q}_{1}$ reduction, sought to address this issue [10, 11]. A confident evaluation of the origins of the SQs observed by these studies is difficult because complete EPR spectra were not reported; comparisons of relative intensities were precluded, and the effects of the NADH: $\mathrm{Q}_{1}$ system on the Fe$\mathrm{S}$ cluster EPR spectrum left unclear. These studies reported that treatment with carboxin, antimycin A and myxothiazol (a complex III $\mathrm{Q}_{\mathrm{o}}$ site inhibitor) caused a decrease in total SQ concentration of $\sim 50 \%$ [10], similar to that observed in our AOX-SMP studies. In contrast, they also determined that (even then) complex I SQs were accumulated to $\sim 100 \%$ occupancy, with a substantial proportion attributed to ' $\mathrm{SQ}_{\mathrm{Nx}}$ ', an ill-defined additional putative complex I SQ. The surprisingly high occupancy may be attributed to SQ formation at the flavin by the hydrophilic ubiquinone analogue [18]. The remaining SQ signals were reported to be sensitive to canonical complex I inhibitors such as rotenone and piericidin A during $\mathrm{NADH}: \mathrm{O}_{2}$ turnover. However, complex I inhibitors prevent reduction of the Q-pool, so cannot definitively localise any SQ to complex I. Finally, attempts to identify complex I SQ species in proteoliposomes, a spectroscopically cleaner system, failed to observe more than a minute fraction of the SQs attributed to complex I in SMPs, despite their reasonable catalytic activity and membrane coupling $[12,20]$. This behaviour is consistent with our proposal that the majority of SQ species observed in SMPs do not, in fact, arise from complex I.

\section{Conclusions}

Despite substantial effort, there is currently no consensus on the origins of SQs within the mitochondrial membrane. The inconsistency of the EPR data presented over the past 40 years [13] is not least the result of the complicated nature of SMPs, which has precluded unambiguous assignments of observed SQ signals to individual components within the mitochondrial membrane. Here, we combined a chimeric respiratory chain with advanced pulse EPR spectroscopic techniques to unambiguously assign the major SQ species generated during NADH oxidation in SMPs to respiratory complex III, with an additional minor SQ species resulting from complex II. We conclude that in earlier studies these complex II and III SQs were sometimes mistakenly assigned to complex I, in the absence of the opportunity to conduct, at the time, controlled inhibition studies, pulse EPR measurements, or focused investigations with the native substrate. Although we observed a piericidin Asensitive SQ signal, which is amplified in the presence of $\mathrm{O}_{2}$ and in AOX-SMPs relative to SMPs, and which increases with prolonged turnover, we cannot definitively assign this $g \sim 2$ signal to complex I. The future of this work now lies in isolating any complex I SQ from other $g \sim 2$ signals, to infer mechanistic information on the role of ubiquinone in the complex I energy coupling reaction from its local magnetic environment. This will necessitate conclusive assignment of the SQ signal in antimycin A- and carboxininhibited AOX-SMPs to complex I by using a spectroscopically clean system, such as complex I-AOX containing proteoliposomes.

\section{Methods}

\section{Preparation of AOX-SMPs and EPR samples}

SMPs were prepared using a protocol adapted from [28]. Twenty millilitres of mitochondria $(\sim 50 \mathrm{mg} / \mathrm{mL})$ were defrosted at $4{ }^{\circ} \mathrm{C}$ and divided equally into four $50-\mathrm{mL}$ centrifuge tubes. The volume of each sample was made up to $45 \mathrm{~mL}$ with SMP buffer $(250 \mathrm{mM}$ sucrose, $10 \mathrm{mM}$ Tris- $\left.\mathrm{SO}_{4}(\mathrm{pH} 7.0)\right)$, and the mitochondria were centrifuged at $16,000 \times g\left(16 \mathrm{~min}, 4{ }^{\circ} \mathrm{C}\right)$. The resulting pellets were combined and homogenised in $40 \mathrm{~mL}$ of SMP buffer. The $\mathrm{pH}$ of the homogenised mitochondria suspension was adjusted to $\mathrm{pH} 9.0$ with $2.5 \mathrm{M}$ Tris at $4{ }^{\circ} \mathrm{C}$ and allowed to stand for $10 \mathrm{~min}$, diluted to $180 \mathrm{~mL}$ with SMP buffer, and centrifuged at $48,000 \times g\left(20 \mathrm{~min}, 4^{\circ} \mathrm{C}\right)$. The mitochondria were then washed twice more in SMP buffer and collected by centrifugation at $16,000 \times g$ (16 min, $4{ }^{\circ} \mathrm{C}$ ). In total, $15 \mathrm{mM} \mathrm{MgSO}_{4}$ was added and each $45-\mathrm{mL}$ sample was sonicated at $130 \mathrm{~W}$ for a total of 2.5 $\min (15 \mathrm{~s}$ on, $60 \mathrm{~s}$ off). The sonicated mixture was centrifuged at $36,000 \times g\left(20 \mathrm{~min}, 4^{\circ} \mathrm{C}\right)$ then the supernatant was centrifuged at $120,000 \times g$ for $30 \mathrm{~min}$ to collect the SMPs. The final pellets were suspended and homogenised in approximately $2 \mathrm{~mL}$ of SMP buffer, and $100 \mu \mathrm{L}$ aliquots at $20-30 \mathrm{mg} / \mathrm{mL}$ were frozen at $-20^{\circ} \mathrm{C}$. Protein concentrations were determined using the test-tube variation of the Pierce bicinchoninic acid (BCA) protein assay kit (Thermo Fisher Scientific, UK).

The alternative oxidase (AOX) from Trypanosoma brucei brucei was prepared and incorporated into SMP membranes as described previously [24, 25]. A $100-\mu \mathrm{L}$ sample of SMPs was incubated with the desired quantity of AOX (from a $3-5 \mathrm{mg} / \mathrm{mL}$ stock) on ice for at least $30 \mathrm{~min}$. Inhibitors were added from DMSO stocks to final concentrations of $100 \mu \mathrm{M}$ (with the percentage of DMSO kept constant in order to avoid erroneous interpretation of solvent-induced radical scavenging [48]). A total of $100 \mu \mathrm{L}$ of sample was transferred to a $4.0 \mathrm{~mm}$ (O.D.) quartz EPR tube (Wilmad) and incubated at room temperature for $5 \mathrm{~min}$. EPR samples were prepared at $20-25 \mathrm{mg} / \mathrm{mL}$ of total SMP protein $(\sim 2.0$ $2.5 \mu \mathrm{M}$ complex I based on approximately $10 \%$ 
complex I content in SMPs [28]). To ensure turnover was maintained until freezing, $\mathrm{O}_{2}$ was bubbled through the sample in the EPR tube: samples were placed under $\mathrm{O}_{2}$ flow for $30 \mathrm{~s}$, NADH added to approximately $20 \mathrm{mM}$, and the samples were then mixed under $\mathrm{O}_{2}$ flow for $5 \mathrm{~s}$ before being frozen by rapid immersion in a cold bath (dry ice/acetone) and transferred to liquid $\mathrm{N}_{2}$ for storage.

\section{$\mathrm{NADH}: \mathrm{O}_{2}$ activity assays}

Kinetic assays were performed with a temperaturecontrolled Agilent Cary $100 \mathrm{UV}$-Vis spectrophotometer by following the absorbance of NADH at $340 \mathrm{~nm}$ $\left(\Delta \varepsilon_{340}=6.22 \mathrm{mM}^{-1} \mathrm{~cm}^{-1}\right)$. SMP activity assays were performed in SMP buffer $(250 \mathrm{mM}$ sucrose, $10 \mathrm{mM}$ Tris$\mathrm{SO}_{4}(\mathrm{pH} 7.0)$ ) at $32{ }^{\circ} \mathrm{C}$ using $30-50 \mu \mathrm{g} / \mathrm{mL}$ SMPs and initiated by $200 \mu \mathrm{M}$ NADH. Uncouplers (carbonyl cyanide 3-chlorophenylhydrazone (CCCP) or gramicidin, a mixture of gramicidin A, B, C, and D) were added from DMSO stock solutions to $2 \mu \mathrm{M}$ or $1 \mu \mathrm{M}$, respectively. The rate of NADH oxidation in the presence and absence of uncoupler was used to calculate the respiratory control ratio (RCR). Piericidin A, antimycin A, or ascofuranone were added from DMSO stock solutions at $2 \mu \mathrm{M}$ to inhibit $\mathrm{NADH}: \mathrm{O}_{2}$ turnover.

\section{Succinate:NAD ${ }^{+}$activity assays}

Reverse electron transfer (RET) assays followed the reduction of $\mathrm{NAD}^{+}$by SMPs by monitoring the absorbance at $340 \mathrm{~nm}$. A total of $50-100 \mu \mathrm{g} / \mathrm{mL}$ SMPs were added to SMP buffer at $32{ }^{\circ} \mathrm{C}$. Forward electron transfer was inhibited with $2 \mu \mathrm{M}$ antimycin A (SMPs) or by anaerobic preparation (AOX-SMPs). In total, $1 \mathrm{mM} \mathrm{NAD}^{+}$ was added and the assay mixture incubated for $1 \mathrm{~min}$. Then $1 \mathrm{mM} \mathrm{MgATP}$ and $10 \mathrm{mM}$ succinate were added to initiate RET. The rate of RET was determined as the maximum rate following succinate addition. Gramicidin $(5 \mu \mathrm{g} / \mathrm{mL})$ or piericidin $\mathrm{A}(2 \mu \mathrm{M})$ was added to inhibit the RET reaction.

\section{ACMA proton-pumping assays}

Proton-pumping assays were performed using an RF5301PC spectrofluorometer (Shimadzu) and the fluorescent dye 9-Amino-6-Chloro-2-Methoxyacridine (ACMA). The $50 \mu \mathrm{g} / \mathrm{mL}$ SMPs/AOX-SMPs were added to a fluorescence cuvette containing ACMA buffer $\left(10 \mathrm{mM}^{\mathrm{T}}\right.$ Tris-SO $\mathrm{S}_{4}$, $50 \mathrm{mM} \mathrm{KCl}(\mathrm{pH} 7.5))$ at $32^{\circ} \mathrm{C}$. ACMA was added at $0.5 \mu \mathrm{M}$ from a DMSO stock solution. Valinomycin was added at $0.1 \mu \mathrm{M}$ to the buffer solution to diminish the membrane potential $(\Delta \psi)$ contribution of $\Delta \mathrm{p}$, allowing $\Delta \mathrm{pH}$ to build to dominate $\Delta \mathrm{p}$. Proton pumping was initiated by the addition of $500 \mu \mathrm{M}$ NADH or $1 \mathrm{mM}$ MgATP. ACMA fluorescence was monitored using an excitation wavelength of $419 \mathrm{~nm}$ and an emission wavelength of 484 $\mathrm{nm}$. Proton gradients were dissipated by addition of piericidin $\mathrm{A}(2 \mu \mathrm{M})$, antimycin $\mathrm{A}(2 \mu \mathrm{M})$, asocfuranone $(2 \mu \mathrm{M})$, alamethicin $(15 \mu \mathrm{g} / \mathrm{mL})$, or oligomycin $(4 \mu \mathrm{M})$.

\section{Measurement of $\Delta \mathrm{p}$ in SMPs and AOX-SMPs}

$\Delta \mathrm{p}$ was measured by varying the potential of NADH:fumarate oxidoreduction during ATP hydrolysis as described previously [28]. Rates were measured in triplicate. The $100 \mu \mathrm{g} / \mathrm{mL}$ SMPs or AOX-SMPs in SMP buffer were treated with $2 \mu \mathrm{M}$ antimycin A (SMPs) or incubated anaerobically (AOX-SMPs [24]) to inhibit respiration before the addition of $1 \mathrm{mM}$ MgATP. Then, catalysis was initiated by addition of $\operatorname{NAD}^{+}(1 \mathrm{mM})$, NADH $(100 \mu \mathrm{M})$, succinate $(500 \mu \mathrm{M})$, and variable concentrations of fumarate $(0.025$ to $40 \mathrm{mM})$. NADH concentrations were monitored at $340 \mathrm{~nm} . \Delta \mathrm{p}$ can be determined by using the different fumarate concentrations to set different potentials via the $\operatorname{NAD}^{+}(-0.335 \mathrm{~V})$ and fumarate $(0.020 \mathrm{~V})$ potentials, as per Eq. 1:

$$
\Delta E=-0.335+0.020-\frac{R T}{2 F} \ln \frac{\{[\mathrm{NADH}][\text { Fumarate }]\}}{\left\{\left[\mathrm{NAD}^{+}\right][\text {Succinate }]\right\}} .
$$

The potential of zero net rate of catalysis, $-2 \Delta E=4 \Delta \mathrm{p}$ (because complex I pumps 4 protons per two electrons), therefore defines $\Delta \mathrm{p}$ [28].

\section{EPR measurements}

EPR measurements were performed using an X/Qband Bruker Elexsys E580 Spectrometer (Bruker BioSpin $\mathrm{GmbH}$, Germany) equipped with a closed-cycle cryostat (Cryogenic Ltd., UK) and X-band split-ring resonator module (ER 4118X-MD5), or a Bruker EMXMicro spectrometer with a helium flow cryostat (Oxford Instruments, UK). All measurements were performed at X-band $(9.7 \mathrm{GHz})$. The magnetic field was calibrated at room temperature with a Bruker strong pitch standard $(g=2.0028)$. Baseline spectra of the empty resonator, of samples containing only buffer, as well as of oxidised complex I were found to be identical; all the CW spectra presented have been baseline subtracted. Continuous-wave EPR measurement conditions were $100 \mathrm{kHz}$ modulation frequency, $7 \mathrm{G}$ modulation amplitude; other measurement conditions are given in figure legends.

\section{EPR simulations}

All spectral simulations were performed using the EasySpin package for MATLAB $[49,50]$. To simulate HYSCORE spectra, ${ }^{14} \mathrm{~N}$ parameters were determined according to the spin Hamiltonian for an electron spin $(S=1 / 2)$ coupled to a nuclear spin with $I=1$, with the energy terms representing the electron Zeeman, nuclear 
Zeeman, hyperfine coupling, and quadrupole coupling, respectively:

$$
\mathcal{H}_{0}=\frac{\beta_{e} g \boldsymbol{B}_{0} S}{\hbar}-\frac{\beta_{n} g_{n} \boldsymbol{B}_{0} \boldsymbol{I}}{\hbar}+S A I+I Q I,
$$

where $\beta_{e}$ is the Bohr magneton, $\beta_{n}$ is the nuclear magneton, $S$ is the electron spin operator (with $S=1 / 2$ ), $I$ is the nuclear spin operator (with $I=1$ for ${ }^{14} \mathrm{~N}$ ), $g$ is the $g$ factor (for the semiquinone taken to be isotropic with $g=2.006), g_{n}$ is the nuclear $g$ factor of ${ }^{14} \mathrm{~N}, B_{0}$ is the static magnetic field vector. $A$ is the hyperfine tensor, with principal components of the diagonalized matrix $A=A_{\mathrm{x}, \mathrm{y}, \mathrm{z}}=A_{\text {iso }}+T$, where $T=[-T,-T, 2 T]$ in axial symmetry, as assumed here. $Q$ is the nuclear quadrupole tensor, for $I=1$ :

$$
\mathrm{Q}=\kappa\left(\begin{array}{lll}
-(1-\eta) & & \\
& -(1+\eta) & \\
& & 2
\end{array}\right),
$$

where $\kappa=\frac{e^{2} q Q}{4 \hbar}$ and $\eta$ are the asymmetry parameter $0<\eta<1$, with 0 designating an axial symmetry and 1 a rhombic symmetry.

Although ${ }^{14} \mathrm{~N}$ nuclei can produce up to 18 cross peaks, the double quantum $\left(\mathrm{dq}_{ \pm}, \mathrm{dq}_{\mp}\right)$ transitions dominate powder HYSCORE spectra here (as is often the case), giving rise to a single pronounced pair of cross peaks. These cross peaks were simulated by varying the values of the hyperfine parameters, $A_{\text {iso }}$ and $T$, and the quadrupole components, $\kappa$ and $\eta$. $A$ was estimated from the HYSCORE frequencies of the double quantum transitions using Eq. 4:

$$
A=\frac{v_{d q+}{ }^{2}-v_{d q-}{ }^{2}}{8_{v I}},
$$

where $v_{I}$ is the nuclear Zeeman frequency. Approximate values of the quadrupole coupling parameters were determined using Eq. 5:

$$
v_{d q \pm}=2\left[v_{e f f \pm}+\kappa^{2}\left(3+\eta^{2}\right)\right]^{\frac{1}{2}}
$$

where $v_{\text {eff }}=\left|v_{I} \pm A / 2\right|$, then refined further to better fit the experimental data.

\section{Supplementary information}

Supplementary information accompanies this paper at https://doi.org/10. 1186/s12915-020-00768-6.

Additional file 1. Adjusting the rate of $\mathrm{NADH}: \mathrm{O}_{2}$ turnover in $\mathrm{AOX}-\mathrm{SMPS}$. Figure S1. Rate matching of AOX-SMPs to SMP NADH:O $\mathrm{O}_{2}$ turnover.

Additional file 2. Comparison of the membrane integrity in SMPs and AOX-SMPS. Figure S2. Comparison of ACMA quenching in SMPs and AOX-SMPs during NADH oxidation and ATP hydrolysis.
Additional file 3. Comparison of EPR signals of reduced SMPs and isolated complex I. Figure S3. X-band CW EPR spectra of reduced SMPS and isolated complex I.

Additional file 4. The microwave power dependence of the $\mathrm{g} \sim 2$ EPR signal in the presence of various inhibitors. Figure S4. Power saturation and relative inhibitor effect on semiquinone signal intensity at $40 \mathrm{~K}$.

Additional file 5. Antimycin A-sensitive ${ }^{14} \mathrm{~N}$ signal in AOX-SMPs. Figure S5. HYSCORE spectrum and subtraction of carboxin inhibited AOX-SMPS.

Additional file 6. Summary of the parameters used to simulate ${ }^{14} \mathrm{~N}$ HYSCORE spectra. Table S1. Hyperfine and quadrupole parameters for ${ }^{14} \mathrm{~N}$ interactions in SMPs and AOX-SMPs.

Additional file 7. HYSCORE spectra of AOX-SMPs at different field positions. Figure S6. Echo-detected field sweep and HYSCORE spectroscopy of oxygen supplemented carboxin- and antimycin Atreated AOX-SMPS.

Additional file 8. Complete HYSCORE spectrum of the $g \sim 2$ signal in complex II inhibited SMPs. Figure S7. Echo-detected field sweep and HYSCORE spectroscopy of oxygen supplemented SMPs treated with carboxin.

Additional file 9. Investigating the 'split N2' signal. Figure S8. The effect of uncouplers on the semiquinone EPR signal in uninhibited SMPs undergoing $\mathrm{NADH}: \mathrm{O}_{2}$ turnover.

\section{Abbreviations}

AOX: Alternative oxidase; EPR: Electron paramagnetic resonance; Fe-S: Ironsulphur cluster; HYSCORE: Hyperfine sublevel correlation; RCR: Respiratory control ratio; SMP: Submitochondrial particles; SQ: Semiquinone

\section{Acknowledgements}

Some of the CW EPR measurements were conducted at the Centre for Advanced ESR (CAESR, University of Oxford, supported by EPSRC EP/ L011972/1), which is gratefully acknowledged for EPR measurement time. Dr. Enrico Salvadori (University of Turin) is acknowledged for helpful discussion of EPR experiments and Dr. Andrew Jones (University of Cambridge) is thanked for assistance with SMP experiments.

\section{Funding}

MMR is grateful to the Leverhulme Trust (research grant number RPG-2018183) and the Royal Society (equipment grant number PGS-R1-191215) for funding. JGF and JH were supported by The Medical Research Council (grant numbers MC_U105663141 and MC_UU_00015/2 to JH).

Availability of data and materials

Additional data related to this publication are freely available at the Imperial College London Research Data Depository, https://doi.org/10.14469/hpc/ 6897 [51].

\section{Authors' contributions}

JJW performed all research and data analysis, except purification of AOX which was performed by JGF. JJW and MMR designed the research with contributions from JH and JGF. MMR and JH directed the research. JJW, $M M R$, and JH wrote the manuscript. All authors read and approved the final manuscript.

\section{Authors' information}

Twitter handle: @MaxieRoessler (Maxie M. Roessler).

Ethics approval and consent to participate Not applicable.

Consent for publication

Not applicable.

Competing interests

The authors declare that they have no competing interests. 


\section{Author details}

${ }^{1}$ School of Biological and Chemical Sciences, Queen Mary University of London, Mile End Road, London E1 4NS, UK. ${ }^{2}$ Medical Research Council Mitochondrial Biology Unit, University of Cambridge, Cambridge CB2 OXY, UK. ${ }^{3}$ Department of Chemistry, Imperial College London, Molecular Sciences Research Hub, White City Campus, Wood Lane, London W12 OBZ, UK.

Received: 4 December 2019 Accepted: 11 March 2020

Published online: 20 May 2020

\section{References}

1. Hirst J. Mitochondrial Complex I. Annu Rev Biochem 2013;82:551-75. https://doi.org/10.1146/annurev-biochem-070511-103700.

2. Friedrich T. On the mechanism of respiratory complex I. J Bioenerg Biomembr. 2014:46:255-68. https://doi.org/10.1007/s10863-014-9566-8.

3. Wirth C, Brandt U, Hunte C, Zickermann V, Zick V. Structure and function of mitochondrial complex I. BBA - Bioenerg. 2016; https://doi.org/10.1016/j. bbabio.2016.02.013.

4. Agip A-NA, Blaza JN, Fedor JG, Hirst J. Mammalian respiratory complex I through the lens of Cryo-EM. Annu Rev Biophys. 2019;48:165-84. https://doi. org/10.1146/annurev-biophys-052118-115704.

5. Gamiz-Hernandez AP, Jussupow A, Johansson MP, Kaila VRI. Terminal Electron-proton transfer dynamics in the quinone reduction of respiratory complex I. J Am Chem Soc. 2017;139:16282-8. https://doi.org/10.1021/jacs. $7 \mathrm{~b} 08486$.

6. Warnau J, Sharma V, Gamiz-Hernandez AP, Di Luca A, Haapanen O, Vattulainen I, et al. Redox-coupled quinone dynamics in the respiratory complex I. Proc Natl Acad Sci. 2018;115:E8413-20. https://doi.org/10.1073/ pnas.1805468115.

7. Kaila VRI. Long-range proton-coupled electron transfer in biological energy conversion: towards mechanistic understanding of respiratory complex I. J R Soc Interface. 2018;15:20170916. https://doi.org/10.1098/rsif.2017.0916.

8. Vinogradov AD, Sled VD, Burbaev DS, Grivennikova VG, Moroz IA, Ohnishi T. Energy-dependent complex l-associated ubisemiquinones in submitochondrial particles. FEBS Lett. 1995;370:83-7. https://doi.org/10. 1016/0014-5793(95)00803-H.

9. Ohnishi T, Johnson JE, Yano T, LoBrutto R, Widger WR. Thermodynamic and EPR studies of slowly relaxing ubisemiquinone species in the isolated bovine heart complex I. FEBS Lett. 2005;579:500-6. https://doi.org/10.1016/j. febslet.2004.11.107.

10. Magnitsky S, Toulokhonova L, Yano T, Sled VD, Hägerhäll C, Grivennikova $V G$, et al. EPR characterization of ubisemiquinones and iron-sulfur cluster N2, central components of the energy coupling in the $\mathrm{NADH}$-ubiquinone oxidoreductase (complex I) in situ. J Bioenerg Biomembr. 2002;34:193-208. https://doi.org/10.1023/a:1016083419979.

11. Yano T, Dunham WR, Ohnishi T. Characterization of the $\Delta \mu_{H}{ }^{+}$-sensitive ubisemiquinone species $\left(\mathrm{SQ}_{\mathrm{Nf}}\right)$ and the interaction with cluster $\mathrm{N} 2$ : new insight into the energy-coupled electron transfer in complex I. Biochemistry. 2005;44:1744-54. https://doi.org/10.1021/bi048132i.

12. Narayanan $M$, Leung $S A$, Inaba $Y$, Elguindy MM, Nakamaru-Ogiso E. Semiquinone intermediates are involved in the energy coupling mechanism of E coli complex I. Biochim Biophys Acta - Bioenerg. 1847;2015:681-9. https://doi.org/10.1016/j.bbabio.2015.04.004.

13. Hirst J, Roessler MM. Energy conversion, redox catalysis and generation of reactive oxygen species by respiratory complex I. Biochim Biophys Acta Bioenerg. 1857;2016:872-83. https://doi.org/10.1016/j.bbabio.2015.12.009.

14. Verkhovskaya $M$, Wikström M. Oxidoreduction properties of bound ubiquinone in complex I from Escherichia coli. Biochim Biophys Acta Bioenerg. 1837;2014:246-50. https://doi.org/10.1016/j.bbabio.2013.11.001.

15. Euro L, Belevich G, Verkhovsky MI, Wikström M, Verkhovskaya M. Conserved lysine residues of the membrane subunit NuoM are involved in energy conversion by the proton-pumping NADH:ubiquinone oxidoreductase (complex I). Biochim Biophys Acta Bioenerg. 2008;1777:1166-72. https://doi. org/10.1016/j.bbabio.2008.06.001.

16. Brandt U. A two-state stabilization-change mechanism for proton-pumping complex I. Biochim Biophys Acta Bioenerg. 1807;2011:1364-9. https://doi. org/10.1016/j.bbabio.2011.04.006.

17. Baradaran R, Berrisford JM, Minhas GS, Sazanov LA. Crystal structure of the entire respiratory complex I. Nature. 2013;494:443-8. https://doi.org/10.1038/ nature11871.
18. King MS, Sharpley MS, Hirst J. Reduction of hydrophilic ubiquinones by the flavin in mitochondrial NADH:ubiquinone oxidoreductase (complex I) and production of reactive oxygen species. Biochemistry. 2009;48:2053-62. https://doi.org/10.1021/bi802282h.

19. Ohnishi T, Sled VD, Yano T, Yagi T, Burbaev DS, Vinogradov AD. Structurefunction studies of iron-sulfur clusters and semiquinones in the NADH-Q oxidoreductase segment of the respiratory chain. Biochim Biophys Acta Bioenerg. 1998;1365:301-8. https://doi.org/10.1016/S0005-2728(98)00082-6.

20. Ohnishi T, Ohnishi ST, Shinzawa-lto K, Yoshikawa S. Functional role of coenzyme $\mathrm{Q}$ in the energy coupling of $\mathrm{NADH}-\mathrm{CoQ}$ oxidoreductase (complex I): stabilization of the semiquinone state with the application of inside-positive membrane potential to proteoliposomes. BioFactors. 2008;32: 13-22. https://doi.org/10.1002/biof.5520320103.

21. Gavrikova EV, Grivennikova VG, Borisov VB, Cecchini G, Vinogradov AD. Assembly of a chimeric respiratory chain from bovine heart submitochondrial particles and cytochrome bd terminal oxidase of Escherichia coli. FEBS Lett. 2009:583:1287-91. https://doi.org/10.1016/j.febslet.2009.03.022.

22. Hastings SF, Kaysser TM, Jiang F, Salerno JC, Gennis RB, Ingledew WJ. Identification of a stable semiquinone intermediate in the purified and membrane bound ubiquinol oxidase-cytochrome bd from Escherichia coli. Eur J Biochem. 1998;255:317-23. https://doi.org/10.1046/j.1432-1327.1998.2550317.x.

23. Hastings SF, Ingledew WJ. A study of the stabilization of semiquinones by the Escherichia coli quinol oxidase cytochrome bd. Biochem Soc Trans. 1996; 24:131-2. https://doi.org/10.1042/bst0240131.

24. Fedor JG, Hirst J. Mitochondrial supercomplexes do not enhance catalysis by quinone channeling. Cell Metab. 2018;28:525-531.e4. https://doi.org/10. 1016/j.cmet.2018.05.024.

25. Jones AJY, Blaza JN, Bridges HR, May B, Moore AL, Hirst J. A self-assembled respiratory chain that catalyzes $\mathrm{NADH}$ oxidation by ubiquinone-10 cycling between complex I and the alternative oxidase. Angew Chemie Int Ed. 2016;55:728-31. https://doi.org/10.1002/anie.201507332.

26. Moore AL, Carré JE, Affourtit C, Albury MS, Crichton PG, Kita K, et al. Compelling EPR evidence that the alternative oxidase is a diiron carboxylate protein. Biochim Biophys Acta Bioenerg. 2008;1777:327-30. https://doi.org/ 10.1016/j.bbabio.2008.01.004.

27. Moore AL, Shiba T, Young L, Harada S, Kita K, Ito K. Unraveling the heater: new insights into the structure of the alternative oxidase. Annu Rev Plant Biol. 2013;64:637-63. https://doi.org/10.1146/annurev-arplant-042811-105432.

28. Pryde KR, Hirst J. Superoxide is produced by the reduced flavin in mitochondrial complex I. J Biol Chem. 2011;286:18056-65. https://doi.org/10. 1074/jbc. M110.186841.

29. Jones AJY, Blaza JN, Varghese F, Hirst J. Respiratory complex I in Bos taurus and Paracoccus denitrificans pumps four protons across the membrane for every NADH oxidized. J Biol Chem. 2017;292:4987-95. https://doi.org/10. 1074/jbc. M116.771899.

30. Van Belzen R, Kotlyar AB, Moon N, Dunham WR, Albracht SPJ. The ironsulfur clusters 2 and ubisemiquinone radicals of NADH:ubiquinone oxidoreductase are involved in energy coupling in submitochondrial particles. Biochemistry. 1997;36:886-93. https://doi.org/10.1021/bi9612982.

31. Sled VD, Rudnitzky NI, Ohnishi T, Hatefi Y. Thermodynamic analysis of flavin in mitochondrial NADH:ubiquinone oxidoreductase (complex I). Biochemistry. 1994;33:10069-75.

32. Ohnishi ST, Shinzawa-Itoh K, Ohta K, Yoshikawa S, Ohnishi T. New insights into the superoxide generation sites in bovine heart NADH-ubiquinone oxidoreductase (Complex I): the significance of protein-associated ubiquinone and the dynamic shifting of generation sites between semiflavin and semiquinone radicals. Biochim Biophys Acta. 2010;1797: 1901-9. https://doi.org/10.1016/j.bbabio.2010.05.012.

33. Beinert H. Copper A of cytochrome c oxidase, a novel, long-embattled, biological electron-transfer site. Eur J Biochem. 1997;245:521-32.

34. Svistunenko DA, Davies N, Brealey D, Singer M, Cooper CE. Mitochondrial dysfunction in patients with severe sepsis: an EPR interrogation of individual respiratory chain components. Biochim Biophys Acta Bioenerg. 2006;1757: 262-72. https://doi.org/10.1016/j.bbabio.2006.03.007.

35. Ruzicka FJ, Beinert H, Schepler KL, Dunham R. SRH. Interaction of ubisemiquinone with a paramagnetic component in heart tissue. Proc Natl Acad Sci U S A. 1975;72:2886-90.

36. Kolling DRJ, Samoilova RI, Holland JT, Berry EA, Dikanov SA, Crofts AR. Exploration of ligands to the $\mathrm{Q}_{i}$ site semiquinone in the $b c_{1}$ complex using high-resolution EPR. J Biol Chem. 2003;278:39747-54. https://doi.org/10. 1074/jbc. M305913200. 
37. Grimaldi S, Arias-Cartin R, Lanciano P, Lyubenova S, Endeward B, Prisner TF, et al. Direct evidence for nitrogen ligation to the high stability semiquinone intermediate in Escherichia coli nitrate Reductase a. J Biol Chem. 2010;285: 179-87. https://doi.org/10.1074/jbc. M109.060251.

38. Maly T, Grgic L, Zwicker K, Zickermann V, Brandt U, Prisner T. Cluster N1 of complex I from Yarrowia lipolytica studied by pulsed EPR spectroscopy. JBIC J Biol Inorg Chem. 2006;11:343-50. https://doi.org/10.1007/s00775-006-0081-1.

39. Kotlyar AB, Sled VD, Burbaev DS, Moroz IA, Vinogradov AD. Coupling site I and the rotenone-sensitive ubisemiquinone in tightly coupled submitochondrial particles. FEBS Lett. 1990;264:17-20.

40. Miki T, Yu L, Yu C-A. Characterization of ubisemiquinone radicals in succinate-ubiquinone reductase. Arch Biochem Biophys. 1992;293:61-6. https://doi.org/10.1016/0003-9861(92)90365-4.

41. Salerno J, Ohnishi T. Studies on the stabilized ubisemiquinone species in the succinate-cytochrome $\mathrm{c}$ reductase segment of the intact mitochondrial membrane system. Biochem J. 1980;192:769-81.

42. Robertson DE, Prince RC, Bowyer JR, Matsuura K, Dutton PL, Ohnishi T. Thermodynamic properties of the semiquinone and its binding site in the ubiquinol: cytochrome c (c2) oxidoreductase of respiratory and photosynthetic systems. J Biol Chem. 1984;259:1758-63.

43. Pietras R, Sarewicz M, Osyczka A. Distinct properties of semiquinone species detected at the ubiquinol oxidation $\mathrm{Q}_{0}$ site of cytochrome $b c_{1}$ and their mechanistic implications. J R Soc Interface. 2016;13:20160133. https://doi. org/10.1098/rsif.2016.0133.

44. Dikanov SA, Samoilova RI, Kolling DRJ, Holland JT, Crofts AR. Hydrogen bonds involved in binding the $\mathrm{Q}_{-}-$site semiquinone in the $b c_{1}$ complex, identified through deuterium exchange using pulsed EPR. J Biol Chem. 2004;279:15814-23. https://doi.org/10.1074/jbc. M313417200.

45. Sarewicz M, Bujnowicz $Ł$, Bhaduri S, Singh SK, Cramer WA, Osyczka A. Metastable radical state, nonreactive with oxygen, is inherent to catalysis by respiratory and photosynthetic cytochromes $b c_{1} / b_{6} f$. Proc Natl Acad Sci. 2017:201618840. https://doi.org/10.1073/pnas.1618840114.

46. Pintscher S, Pietras R, Sarewicz M, Osyczka A. Electron sweep across four bhemes of cytochrome $b c_{1}$ revealed by unusual paramagnetic properties of the $\mathrm{Q}_{j}$ semiquinone intermediate. Biochim Biophys Acta Bioenerg. 1859;2018:459-69.

47. Ohnishi T, Ohnishi ST, Shinzawa-ltoh K, Yoshikawa S, Weber RT. EPR detection of two protein-associated ubiquinone components ( $\mathrm{SQ}_{\mathrm{Nf}}$ and $\mathrm{SQ}_{\mathrm{NS}}$ ) in the membrane in situ and in proteoliposomes of isolated bovine heart complex I. Biochim Biophys Acta. 1817;2012:1803-9. https://doi.org/10. 1016/j.bbabio.2012.03.032.

48. Phillis JW, Estevez AY, O'Regan MH. Protective effects of the free radical scavengers, dimethyl sulfoxide and ethanol, in cerebral ischemia in gerbils. Neurosci Lett. 1998;244:109-11.

49. Stoll S, Schweiger A. EasySpin, a comprehensive software package for spectral simulation and analysis in EPR. J Magn Reson. 2006;178:42-55. https://doi.org/10.1016/j.jmr.2005.08.013.

50. Stoll S, Britt RD. General and efficient simulation of pulse EPR spectra. Phys Chem Chem Phys. 2009;11:6614

51. Wright JJ, Fedor JG, Hirst J, Roessler MM. Using a chimeric respiratory chain and EPR spectroscopy to determine the origin of semiquinone species previously assigned to mitochondrial complex I. Data deposition. 2020. Imperial College London Research Data Depository. https://doi.org/10. 14469/hpc/6897.

\section{Publisher's Note}

Springer Nature remains neutral with regard to jurisdictional claims in published maps and institutional affiliations.

Ready to submit your research? Choose BMC and benefit from:
- fast, convenient online submission
- thorough peer review by experienced researchers in your field
- rapid publication on acceptance
- support for research data, including large and complex data types
- gold Open Access which fosters wider collaboration and increased citations
- maximum visibility for your research: over 100M website views per year
At BMC, research is always in progress.
Learn more biomedcentral.com/submissions

